\title{
Interações entre fármacos e medicamentos fitoterápicos à base de ginkgo ou ginseng
}

\author{
Rodrigo F. Alexandre, Fabíola Bagatini, Cláudia M. O. Simões* \\ Laboratório de Farmacognosia, Departamento de Ciências Farmacêuticas, Universidade Federal de Santa \\ Catarina, Campus Trindade, 88040-900 Florianópolis-SC, Brasil
}

\begin{abstract}
RESUMO: Os medicamentos fitoterápicos são amplamente utilizados, principalmente, pelos portadores de doenças crônicas e em associações medicamentosas com diversos fármacos. As possíveis interações entre eles estão sendo muito estudadas, pois podem alterar os perfis de eficácia e segurança de muitos fármacos. Nesta revisão, as informações foram localizadas, avaliadas e sistematizadas e contêm as principais interações entre fármacos e medicamentos fitoterápicos elaborados com ginkgo ou ginseng. Verificou-se que os medicamentos fitoterápicos elaborados com tais plantas podem interferir na farmacocinética e/ou farmacodinâmica de diversos fármacos, podendo provocar conseqüências graves aos pacientes. O ginkgo pode interferir com anticoagulantes orais, antiplaquetários e com fármacos metabolizados pelo sistema P450-CYP3A4. O ginseng pode interagir com antidepressivos inibidores da monoamino oxidase, anticoagulantes orais, anti-hipertensivos, e contraceptivos à base de estrogênios. Além disso, não é recomendada a administração concomitante de ginkgo ou ginseng com antineoplásicos. Nesse sentido, o uso concomitante de medicamentos fitoterápicos à base de ginkgo ou ginseng com outros fármacos deve ser adequadamente monitorado.
\end{abstract}

Unitermos: Ginkgo biloba, Panax ginseng, ginkgo, ginseng, interações medicamentosas.

\begin{abstract}
Interactions between drugs and ginkgo or ginseng herbal medicines". Herbal medicines are widely used especially by patients with chronic diseases, often administered concomitantly with synthetic drugs, raising the potential of pharmacokinetic and pharmacodynamic drug-herb interactions. From the last years, there is an increasing interest in this subject reflected by the great number of documented case reports, in vivo studies, and also clinical trials evaluating drug-herb interactions. The aim of this paper was to review the literature in order to identify reported interactions between ginkgo or ginseng herbal medicines and drugs as well as to evaluate and summarize these information. Interactions between ginkgo or ginseng herbal medicines and drugs can occur and may lead to serious consequences. Ginkgo has the potential to cause significant interactions with anticoagulant and antiplatelet drugs, and also with drugs metabolized by the cytochrome P450 enzyme system, especially by CYP3A4. Ginseng has the potential to cause significant interactions with monoamine oxidase inhibitors, warfarin, antihypertensive agents and estrogens. Additionally, both should be avoided with anticancer drugs. Based on these data and regarding patient's safety, the concomitant use of herbal medicines and drugs has to be properly surveyed by physicians and/or other health care professionals.
\end{abstract}

Keywords: Ginkgo biloba, Panax ginseng, ginkgo, ginseng, drug-herb interactions.

\section{INTRODUÇÃO}

A utilização de plantas medicinais e medicamentos fitoterápicos para a recuperação da saúde é uma prática generalizada, sendo o resultado do acúmulo secular de conhecimentos empíricos sobre a ação dos vegetais por diversos grupos étnicos (Simões et al., 1986). Diversos estudos mostram que os medicamentos fitoterápicos são amplamente comercializados em muitos países, principalmente, EUA (Barnes et al., 2004; Blumenthal et al., 2006), Europa (Menniti-Ippolito et al., 2002; Hartel \& Volger, 2004; De Smet, 2005) e Brasil (Momesso, 2002; Ribeiro et al., 2005; Silva et al., 2006). Os usuários de plantas medicinais e/ou medicamentos fitoterápicos são, predominantemente, pessoas adultas e idosas, que utilizam outros medicamentos como tratamento principal de doenças crônicas (MacLennan et al., 1996) e, geralmente, acreditam que a fitoterapia é uma alternativa terapêutica isenta de efeitos adversos e/ou incapaz de causar interações medicamentosas (Ernst et al., 1995). Muitas vezes, o uso desses recursos é estimulado de maneira pouco criteriosa. Os conhecimentos empíricos acumulados no passado (tradição cultural) e os científicos desenvolvidos, ao longo do tempo, principalmente com a condução de ensaios clínicos randomizados, mostram que as plantas medicinais e os medicamentos fitoterápicos podem, também, provocar efeitos adversos, toxicidade e 
apresentar contra-indicações de uso (Alexandre et al., 2005a,b).

As plantas medicinais e, por conseqüência, os medicamentos fitoterápicos são constituídos de misturas complexas de muitos compostos químicos, que podem ser responsáveis pela suas ações polivalentes. Essas ações podem ser explicadas pela interdependência única das mesmas, quando efeitos aditivos, antagônicos e/ ou sinérgicos ocorrem como resultado da interação de vários constituintes químicos ativos, em diversos sítios de ação, em diferentes órgãos e tecidos (Williamson, 2005). Contudo, em muitos casos, os constituintes químicos responsáveis pelas atividades farmacológicas das plantas medicinais e/ou medicamentos fitoterápicos são desconhecidos e a complexidade dos constituintes presentes aumenta a possibilidade de ocorrer interações quando fármacos são utilizados concomitantemente (Fugh-Berman \& Ernst., 2001; Mills et al., 2005).

As interações entre fármacos e os componentes químicos presentes nas plantas medicinais e nos medicamentos fitoterápicos podem causar alterações nas concentrações plasmáticas dos fármacos e, conseqüentemente, mudanças nos seus perfis de eficácia e/ou segurança. Essas interações podem ser classificadas em farmacocinéticas e farmacodinâmicas. No primeiro caso, os processos de absorção, distribuição, metabolismo e excreção do fármaco, podem ser afetados, resultando em ampliação ou redução dos efeitos esperados. A maioria dos fármacos tem seus efeitos terapêuticos explicados através da ligação a receptores específicos. A administração concomitante de fármacos e plantas medicinais e/ou medicamentos fitoterápicos pode alterar os níveis de resposta a esses receptores, provocando a ampliação ou redução do efeito farmacológico esperado, devido ao sinergismo ou antagonismo, respectivamente (Fugh-Berman, 2000; Izzo \& Ernst, 2001).

As plantas medicinais e os medicamentos fitoterápicos são amplamente utilizados no Brasil como alternativa terapêutica, principalmente por aqueles que estão em tratamento de doenças crônicas com outros medicamentos. Nesse sentido, o objetivo desse artigo foi realizar um levantamento bibliográfico sobre as principais interações entre fármacos e medicamentos fitoterápicos elaborados à base de ginkgo (Ginkgo biloba L.) e ginseng (Panax ginseng C. A. Mey. e Panax quinquefolius L.), contribuindo, juntamente com outras publicações nacionais (Cordeiro et al., 2005), para a divulgação e prevenção de tais interações.

\section{METODOLOGIA}

De acordo com a literatura, ginkgo e ginseng são plantas utilizadas para a elaboração de medicamentos fitoterápicos amplamente utilizados na Europa, EUA (Blumenthal et al., 2006; van den Bout-van den Beukel et al., 2006) e em todo o território nacional (Cordeiro et al., 2005).
Realizou-se um levantamento bibliográfico para a busca de informações sobre as possíveis interações do ginkgo ou ginseng com determinados fármacos. Para isso, foram utilizadas as bases de dados MEDLINE e COCHRANE COLLABORATION, utilizando-se como palavras-chaves os binômios científicos "Ginkgo biloba", "Panax ginseng" e "Panax quinquefolius", sem restrição de data, idioma e tipo de publicação e indexados até agosto de 2006. Essas bases de dados foram utilizadas para a localização dos estudos pré-clínicos, que foram realizados para elucidar os mecanismos envolvidos nas possíveis interações medicamentosas, além de relatos de casos, ensaios clínicos e revisões sistemáticas e/ou meta-análises, realizados para avaliar a segurança dos medicamentos fitoterápicos elaborados com as plantas medicinais em questão. Além disso, foram realizadas buscas manuais de ensaios clínicos nas listas de referências de livros especializados e/ou de artigos já localizados.

\section{RESULTADOS}

Os resultados da pesquisa bibliográfica realizada sobre as possíveis interações entre fármacos e medicamentos fitoterápicos elaborados com ginkgo (Ginkgo biloba L.) e ginseng (Panax ginseng C. A. Mey. e Panax quinquefolius L.) estão apresentados nas Tabelas 1 e 2, respectivamente.

\section{DISCUSSÃO}

\section{Ginkgo}

Os extratos secos padronizados de Ginkgo biloba, principalmente o EGb 761 e o LI 1370, são utilizados para a elaboração de medicamentos fitoterápicos (Hillebrand \& Oliveira, 2004). De acordo com os resultados dos ensaios clínicos randomizados, duplos-cegos e controlados, esses medicamentos são eficazes no tratamento da claudicação intermitente (Pittler \& Ernst, 2000; Jacoby\& Mohler, 2004) e da insuficiência cerebral (doença caracterizada por sintomas típicos, tais como dificuldades de concentração e memória, confusão, indisposição, cansaço, redução da performance física, ansiedade, tontura, zumbido e cefaléia) (Gerhardt et al., 1990; Kleijnen \& Knipschild, 1992; Hopfenmüller, 1994), podendo constituir uma alternativa terapêutica para o tratamento de demências do tipo Alzheimer e multi-infarto (Oken et al., 1998; Rogers et al., 1998; Wettstein, 2000; Le Bars et al., 2002; Schulz, 2003) e do zumbido (Ernst \& Stevinson, 1999; Drew \& Davies, 2001; Morgenstern \& Biermann, 2002; Rejali et al., 2004; Smith et al., 2005). Os constituintes químicos considerados ativos do ginkgo são os flavonóides (glicosídeos de quercetina, canferol e isoramnetina) e as lactonas terpênicas (ginkgolídeos e bilobalídeo) (DeFeudis, 1998). 
A Comissão E alemã afirma que não há interações de relevância clínica entre medicamentos fitoterápicos elaborados com ginkgo e fármacos (Blumenthal et al., 2000). No entanto, existem vários relatos de casos sobre suas prováveis interações com fármacos anticoagulantes orais, antiplaquetários, antiinflamatórios não-esteroidais, anticonvulsivantes, antidepressivos, anti-hipertensivos e antiulcerosos (Tabela 1). Após a tabela, tais interações estão descritas em maiores detalhes, finalizando com algumas considerações relativas aos dados encontrados na literatura. hematoma subfrênico e hemorragia vítreos foi relatado após o uso concomitante de ácido acetilsalicílico e ginkgo (Rosenblatt \& Mindel, 1997). Recentemente, foi publicado o relato do caso de uma mulher de 77 anos, que teve sangramento pós-operatório persistente e que ingeria, concomitantemente, um medicamento elaborado com ginkgo para o tratamento de deficiência cognitiva e ácido acetilsalicílico. Este quadro clínico foi controlado após a interrupção do tratamento com o medicamento fitoterápico (Bebbington et al., 2005).

Tabela 1. Possíveis interações entre medicamentos fitoterápicos à base de ginkgo (Ginkgo biloba L.) e fármacos.

\begin{tabular}{|c|c|c|c|c|}
\hline Classe farmacológica & Fármaco & $\begin{array}{l}\text { Mecanismo de } \\
\text { interação }\end{array}$ & Possíveis efeitos & Referências \\
\hline $\begin{array}{l}\text { Anticoagulantes orais } \\
\text { e Antiplaquetários }\end{array}$ & Varfarina & $\begin{array}{l}\text { Ginkgolídeo B pode } \\
\text { inibir o fator de } \\
\text { ativação plaquetária } \\
\text { inibindo a sua } \\
\text { agregação }\end{array}$ & $\begin{array}{l}\uparrow \text { risco de } \\
\text { hemorragia }\end{array}$ & $\begin{array}{l}\text { Rosenblatt \& Mindel, 1997; } \\
\text { Matthews, 1998; Lambrecht et al., } \\
\text { 2000; Bebbington et al., } 2005\end{array}$ \\
\hline $\begin{array}{l}\text { Antiinflamatórios } \\
\text { não-esteroidais }\end{array}$ & $\begin{array}{l}\text { Ibuprofeno } \\
\text { Ácido } \\
\text { acetilsalicílico }\end{array}$ & $\begin{array}{l}\text { Ginkgolídeo B pode } \\
\text { inibir o fator de } \\
\text { ativação plaquetária } \\
\text { inibindo a sua } \\
\text { agregação }\end{array}$ & $\begin{array}{l}\uparrow \text { risco de } \\
\text { hemorragia }\end{array}$ & $\begin{array}{l}\text { Meisel et al., 2003; Varona \& } \\
\text { Morales, } 2005\end{array}$ \\
\hline 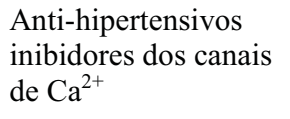 & Nifedipina & $\begin{array}{l}\text { Inibição da isoforma } \\
\text { CYP3A4 }\end{array}$ & $\begin{array}{l}\uparrow \text { efeitos adversos } \\
\text { do fármaco }\end{array}$ & $\begin{array}{l}\text { Smith et al., 2001; Yoshioka et } \\
\text { al., } 2004\end{array}$ \\
\hline $\begin{array}{l}\text { Antiulcerosos } \\
\text { inibidor da bomba de } \\
\text { prótons }\end{array}$ & Omeprazol & $\begin{array}{l}\text { Indução das } \\
\text { isoformas CYP2C19 } \\
\text { e CYP3A4 } \\
\end{array}$ & $\begin{array}{l}\downarrow[] \text { plasmática e } \\
\text { do efeito } \\
\text { terapêutico }\end{array}$ & Yin et al., 2004 \\
\hline Anticonvulsivantes & $\begin{array}{l}\text { Valproato de } \\
\text { sódio }\end{array}$ & $\begin{array}{l}\text { Ainda não } \\
\text { estabelecido }\end{array}$ & $\begin{array}{l}\downarrow \text { eficácia do } \\
\text { fármaco }\end{array}$ & Granger, 2001 \\
\hline Antipsicóticos & $\begin{array}{l}\text { Haloperidol } \\
\text { Olanzapina }\end{array}$ & $\begin{array}{l}\text { Seqüestro dos } \\
\text { radicais livres } \\
\text { produzidos pela } \\
\text { atividade } \\
\text { hiperdopaminérgica }\end{array}$ & $\begin{array}{l}\uparrow \text { dos efeitos } \\
\text { terapêuticos }\end{array}$ & $\begin{array}{l}\text { Zhou et al., 1999; Zhang et al., } \\
\text { 2001a,b; Atmaca et al., } 2005\end{array}$ \\
\hline Ansiolíticos & Alprazolam & $\begin{array}{l}\text { Não há alteração das } \\
\text { isoformas CYP 2D6 } \\
\text { e 3A4 }\end{array}$ & - & Markowitz et al., 2003 \\
\hline \multirow{2}{*}{ Antidepressivos } & Trazodona & $\begin{array}{l}\text { Indução da isoforma } \\
\text { CYP3A4 }\end{array}$ & $\begin{array}{l}\uparrow \text { efeitos sedativos } \\
\text { podendo provocar } \\
\text { coma }\end{array}$ & Galluzzi et al., 2000 \\
\hline & Fluoxetina & $\begin{array}{l}\uparrow[] \text { de óxido nítrico } \\
\text { na musculatura } \\
\text { peniana }\end{array}$ & $\begin{array}{l}\text { Redução da } \\
\text { disfunção sexual }\end{array}$ & $\begin{array}{l}\text { Cohen \& Bartlik, 1998; Kang et } \\
\text { al., 2002; Wheatley, } 2004\end{array}$ \\
\hline
\end{tabular}

\section{Anticoagulantes orais e antiplaquetários}

O uso concomitante de medicamentos fitoterápicos à base de ginkgo com anticoagulantes e/ou antiplaquetários pode aumentar o risco de complicações hemorrágicas, já que estes medicamentos aumentam a fluidez sangüínea (DeFeudis, 1998). Um paciente de 78 anos desenvolveu hemorragia intracerebral após uso concomitante de varfarina e de um medicamento fitoterápico à base de ginkgo (Matthews, 1998; Lambrecht et al., 2000). Um caso de hifema espontâneo,

\section{Antiinflamatórios não-esteroidais}

Há um relato de caso de hemorragia cerebral fatal ocorrido em um homem idoso, após o uso do ginkgo durante mais de dois anos para o tratamento de tonturas ocasionais, e ibuprofeno, durante quatro semanas, para o tratamento da osteoartrite. Neste caso, o paciente não apresentava, aparentemente, fator de risco para o sangramento intracraniano. Tal associação pode aumentar a incidência de sangramentos, provavelmente pela inibição do tromboxano $\mathrm{A}_{2}$, que é um componente 
da cascata de coagulação (Meisel et al., 2003).

Recentemente, foram relatados dois casos de hemorragia cerebral em pacientes idosos e portadores da doença de Alzheimer tratados com extratos de ginkgo e ácido acetilsalicílico, durante aproximadamente dois anos. Nos dois casos, o quadro clínico foi recuperado após a suspensão do antiinflamatório (Varona \& Morales, 2005). Estes casos podem ser explicados pelo fato de que os ginkgolídeos A e B, presente no ginkgo, é um inibidor do fator de agregação plaquetária (Prehn \& Krieglstein, 1993).

Além disso, o uso concomitante de preparações contendo ginkgo com outros atiinflamatórios nãoesteroidais pode potencializar os riscos de sangramentos gastrointestinais (Micromedex, 2005).

\section{Anti-hipertensivos}

O uso concomitante de medicamentos fitoterápicos à base de ginkgo e nifedipina (antagonista dos canais de cálcio) pode aumentar a freqüência de efeitos adversos desse anti-hipertensivo, tais como cefaléia, rubor e edema de tornozelo (Micromedex, 2005). De acordo com um ensaio clínico aberto, o uso do extrato de ginkgo provocou um aumento médio na concentração plasmática da nifedipina de, aproximadamente, $29 \%$ após 30 min da sua administração (Smith et al., 2001). O provável mecanismo de interação é a inibição da isoforma CYP3A4 do sistema enzimático citocromo $\mathrm{P} 450$ pelos componentes químicos do ginkgo (Micromedex, 2005). Em outro ensaio clínico randomizado realizado com oito voluntários saudáveis, verificou-se que a concentração plasmática máxima da nifedipina em dois voluntários foi duplicada após o seu uso concomitante com um medicamento fitoterápico elaborado com ginkgo, provocando cefaléia intensa, desmaio, rubor intenso e taquicardia (Yoshioka et al., 2004).

\section{Antiulcerosos}

O omeprazol é um fármaco utilizado no tratamento da úlcera péptica e do refluxo gastroesofágico, sendo que a sua metabolização ocorre, principalmente, via isoforma CYP2C19 do sistema hepático P450. Através de um ensaio clínico randomizado conduzido com 18 voluntários sadios, verificou-se que o ginkgo, quando administrado concomitantemente com este fármaco, reduziu a biodisponibilidade do mesmo e aumentou a concentração plasmática do seu metabólito ativo, denominado 5-hidróxiomeprazol. Esse resultado indica que o ginkgo pode induzir a isoforma CYP2C19 e, com isso, reduzir a concentração plasmática do omeprazol (Yin et al., 2004).

\section{Anticonvulsivantes}

O uso de medicamentos fitoterápicos à base de ginkgo pode reduzir a eficácia dos anticonvulsivantes. Há um relato de dois pacientes, com epilepsia previamente bem controlada com valproato de sódio, que apresentaram crises epilépticas após a ingestão de um medicamento à base de extrato de ginkgo, cuja elaboração não foi detalhada pelos autores. As crises foram controladas após a suspensão do tratamento com o medicamento fitoterápico (Granger, 2001).

\section{Antipsicóticos}

A associação de medicamentos fitoterápicos à base de ginkgo pode aumentar a atividade do haloperidol e da olanzapina, provavelmente, devido ao efeito antioxidante do ginkgo. Esta interação foi estudada em quatro ensaios clínicos que verificaram o aumento da atividade terapêutica do haloperidol $(0,25$ $\mathrm{mg} / \mathrm{kg} / \mathrm{dia}$ ) (Zhou et al., 1999; Zhang et al., 2001a,b) e da olanzapina (5 a $20 \mathrm{mg} / \mathrm{dia}$ ) (Atmaca et al., 2005), em portadores de esquizofrenia. Dois destes estudos verificaram a redução dos níveis plasmáticos da enzima superóxido dismutase (Zhou et al., 1999; Zhang et al., 2001a) e um terceiro estudo verificou, além da redução da superóxido dismutase, a redução da catalase (Atmaca et al., 2005), sugerindo que o ginkgo seqüestra os radicais livres produzidos pela atividade hiperdopaminérgica, característica nos casos de esquizofrenia.

\section{Ansiolíticos}

Um ensaio clínico avaliou a possível alteração da atividade das isoformas CYP 2D6 e 3A4 do citocromo P450 hepático, após o tratamento com o extrato de ginkgo (EGB 761), em 12 voluntários saudáveis. Para isso, foram administrados fármacos metabolizados por tais isoformas (dextrometorfano e alprazolam, respectivamente), juntamente com $240 \mathrm{mg} / \mathrm{dia}$ do medicamento fitoterápico, durante 14 dias. Verificou-se que o extrato de ginkgo não modificou a farmacocinética do dextrometorfano, indicando que não há alteração da isoforma CYP 2D6. Apesar da redução em 17\% na área sobre a curva concentração versus efeito do alprazolam, o extrato de ginkgo também não apresentou interação com a isoforma CYP 3A4 (Markowitz et al., 2003).

\section{Antidepressivos}

Está descrito na literatura o relato do caso de um paciente com doença de Alzheimer, que entrou em coma após o uso de um medicamento fitoterápico à base de ginkgo e de trazodona (antidepressivo atípico), que foi revertido com a administração de flumazenil. Essa interação pode ser explicada pelo fato de que os flavonóides presentes no ginkgo podem aumentar a atividade gabaérgica, devido ao efeito direto nos receptores benzodiazepínicos (Sasaki et al., 1999). 
Além disso, o ginkgo pode aumentar a atividade da isoforma CYP3A4 do sistema microssomal P450, elevando a concentração plasmática do metabólito ativo da trazodona (Galluzzi et al., 2000).

A disfunção sexual é um efeito adverso comum dos antidepressivos inibidores da recaptação de serotonina.Alguns estudos mostraram que medicamentos elaborados com Ginkgo biloba podem contribuir para a redução de tais efeitos adversos, principalmente, porque o ginkgo pode aumentar a liberação de óxido nítrico na musculatura peniana (Cohen \& Bartlik, 1998; Kang et al., 2002; Wheatley, 2004). Porém, os resultados foram contraditórios e sem significância estatística e clínica devido aos graves problemas metodológicos apresentados nestes estudos, tais como a falta de grupos controle, o emprego de extratos não padronizados, a heterogeneidade das amostras, entre outros.

Em suma, os estudos disponíveis sugerem uma potencial interação entre os medicamentos fitoterápicos elaborados com ginkgo e anticoagulantes orais, antiplaquetários e com fármacos metabolizados pelo sistema P450 CYP3A4. Portanto, deve-se monitorar o tempo de pró-trombina em pacientes tratados com ginkgo e varfarina, e acompanhar clinicamente aqueles que ingerem ginkgo, concomitantemente com ácido acetilsalicílico, alprazolam e trazodona. Como os antineoplásicos são metabolizados, principalmente, pelas isoformas CYP3A4, deve-se evitar sua utilização concomitante com o ginkgo (Sparreboom et al., 2004).

\section{Ginseng}

Muitas preparações à base de ginseng disponíveis comercialmente apresentam outros constituintes químicos na formulação, impedindo uma avaliação precisa da eficácia e da segurança desta planta presente nesses medicamentos. Os ensaios clínicos randomizados, duplos-cegos e controlados mostraram que o extrato seco de Panax ginseng G115 pode ser uma alternativa terapêutica na melhora da performance física, psicomotora e cognitiva, e também como imunomodulador (Vogler et al., 1999; Bucci, 2000; Coleman et al., 2003). Os seus constituintes químicos considerados ativos são as saponinas triterpênicas tetracíclicas e pentacíclicas (Liu \& Xiao, 1992).

Um estudo in vitro mostrou que as saponinas do ginseng inibiram, de maneira concentração-dependente, a atividade do citocromo $\mathrm{P} 450$ dos microssomos hepáticos isolados de ratos. $\mathrm{O}$ efeito inibitório foi evidente na oxidação do $p$-nitrofenol mediada pela CYP2E1 e na N-metilação da eritromicina mediada pela CYP3A (Kim et al., 1997). Porém, os efeitos do ginseng sobre esse sistema metabolizador de xenobióticos, em humanos, ainda não foram totalmente elucidados (Ionnides, 2002). Outros pesquisadores mostraram que o ginseng não provoca efeitos relevantes sobre o metabolismo de fármacos mediado pelo sistema CYP, em humanos, apresentando menor probabilidade de

Tabela 1. Possíveis interações entre medicamentos fitoterápicos à base de ginseng (Panax ginseng C. A. Mey. e Panax quinquefolius L.) e fármacos.

\begin{tabular}{|c|c|c|c|c|}
\hline Classe farmacológica & Fármacos & $\begin{array}{l}\text { Mecanismo de } \\
\text { interação }\end{array}$ & Possíveis efeitos & Referências \\
\hline $\begin{array}{l}\text { Antidepressivos } \\
\text { inibidores da MAO }\end{array}$ & Fenelzina & Não estabelecido & $\begin{array}{l}\text { Cefaléia, insônia e } \\
\text { tremor }\end{array}$ & $\begin{array}{l}\text { Shader \& Greenblatt, 1985; } \\
\text { Shader \& Greenblatt, 1988; } \\
\text { Jones \& Runikis, } 1987\end{array}$ \\
\hline Anticoagulantes orais & Varfarina & $\begin{array}{l}\text { Potencialização do } \\
\text { efeito } \\
\text { anticoagulante }\end{array}$ & $\begin{array}{l}\uparrow \text { risco de } \\
\text { hemorragia }\end{array}$ & $\begin{array}{l}\text { Janetzky \& Morreale, 1997; } \\
\text { Yuan et al., 2004;Jiang et al., } \\
\text { 2004; Jiang et al., } 2005\end{array}$ \\
\hline Estrogênios & $\begin{array}{l}\text { Contraceptivos } \\
\text { orais à base de } \\
\text { estrogênios }\end{array}$ & $\begin{array}{l}\text { Antividade } \\
\text { estrogênica } \\
\text { sinérgica }\end{array}$ & $\begin{array}{l}\text { Mastalgia, } \\
\text { sangramento } \\
\text { menstrual excessivo }\end{array}$ & $\begin{array}{l}\text { Palmer et al., 1978; Punnonen \& } \\
\text { Lukola, 1980; Greenspan, } 1983\end{array}$ \\
\hline 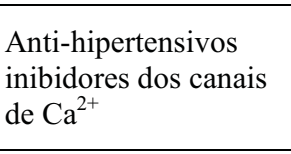 & Nifedipina & Não estabelecido & $\begin{array}{l}\text { Cefaléia, } \\
\text { constipação, } \\
\text { insuficiência } \\
\text { cardíaca }\end{array}$ & Smith et al., 2001 \\
\hline $\begin{array}{l}\text { Anti-hipertensivos } \\
\text { diuréticos de alça }\end{array}$ & Furosemida & Não estabelecido & Hipotensão e edema & Becker et al., 1996 \\
\hline Hipoglicemiantes & Insulina & $\begin{array}{l}\text { 个 da secreção e } \\
\text { sensibilidade à } \\
\text { insulina }\end{array}$ & Hipoglicemia grave & $\begin{array}{l}\text { Vuksan et al., 2000a,b,c; Vuksan } \\
\text { et al., } 2001\end{array}$ \\
\hline Etanol & - & $\begin{array}{l}\text { Indução da } \\
\text { isoforma CYP2E1 }\end{array}$ & $\begin{array}{l}\text { Redução da } \\
\text { concentração } \\
\text { plasmática do etanol }\end{array}$ & Lee et al., 1987 \\
\hline \multirow[t]{2}{*}{ Vacinas } & $\begin{array}{l}\text { Vacinas para } \\
\text { prevenção da gripe } \\
\text { por influenza }\end{array}$ & $\begin{array}{l}\uparrow \text { da resposta } \\
\text { imunológica }\end{array}$ & $\begin{array}{l}\downarrow \text { dos sintomas da } \\
\text { gripe }\end{array}$ & Scaglione et al., 1996 \\
\hline & & & & $\begin{array}{r}\text { Rev. Bras. Farmacogn. } \\
\text { Braz J. Pharmacogn. } \\
\text { 18(1): Jan./Mar. } 2008\end{array}$ \\
\hline
\end{tabular}


causar interações farmacocinéticas do que outras espécies vegetais (Gurley et al., 2005). Alguns relatos de interações envolvendo medicamentos fitoterápicos à base de ginseng e outros fármacos já foram publicados e estão apresentados na Tabela 2. Após a tabela, tais interações estão descritas em maiores detalhes, finalizando com algumas considerações relativas aos dados encontrados na literatura.

\section{Antidepressivos}

Foram relatados dois casos de interações medicamentosas entre fenelzina (antidepressivo da classe dos inibidores da enzima monoamina oxidase) e medicamentos fitoterápicos elaborados com ginseng. No primeiro caso, uma mulher de 64 anos apresentou cefaléia, insônia e tremores após utilizar um produto à base de ginseng, ginseng na forma de chá e fenelzina. Três anos depois, quando ainda utilizava fenelzina, apresentou os mesmos sintomas, após utilizar cápsulas de ginseng (Shader \& Greenblatt, 1985). Os autores não relataram o desfecho do caso, mas relacionaram o aparecimento desses efeitos devido a interação entre o produto contendo ginseng e a fenelzina (Shader \& Greenblatt, 1988). No segundo caso, uma mulher de 42 anos, com depressão, sem história familiar de mania e/ ou doenças psiquiátricas, iniciou um tratamento com fenelzina $(45 \mathrm{mg} / \mathrm{dia})$ e uma preparação à base de ginseng. Inicialmente, obteve redução dos sintomas da depressão, mas posteriormente, apresentou sintomas de mania, insônia, irritabilidade, cefaléia e alucinações e, por isso, suspendeu os medicamentos antidepressivos. Como houve o aparecimento dos sintomas da depressão, retomou o tratamento com a fenelzina, sem suspender a preparação à base de ginseng e, novamente, apresentou episódios de cefaléia. Os autores relacionaram o aparecimento desses sintomas devido a uma possível interação entre a fenelzina e o ginseng (Jones \& Runikis, 1987). O mecanismo envolvido nesta interação ainda é desconhecido, mas pode estar relacionado com a atividade do ginseng sobre o sistema nervoso central.

\section{Anticoagulantes orais}

Foi relatada a possível interação entre um medicamento fitoterápico à base de $P$. ginseng e varfarina (Janetzky \& Morreale, 1997). Neste caso, uma mulher de 47 anos, que tinha uma válvula mecânica na aorta, tratava-se com varfarina ( $5 \mathrm{mg} /$ dia) há sete anos como anticoagulante, e iniciou o uso de três cápsulas diárias do medicamento fitoterápico. O tempo de pró-trombina, que esteve normal nos últimos nove meses, declinou para 1,5 (normal: 2,5 a 3,5), após duas semanas do início do tratamento com o ginseng. Esse valor retornou ao normal após a suspensão do medicamento fitoterápico, sugerindo uma interação entre os ginsenosídeos e a varfarina. Como estudos em animais não mostraram interferência do ginseng sobre a farmacodinâmica e a farmacocinética da varfarina (Zhu et al., 1999), o significado clínico desse caso não pôde ser estabelecido (Coon \& Ernst, 2002). Com base nestes relatos de casos, foram conduzidos três ensaios clínicos para avaliar a possível interação entre $P$. ginseng e varfarina em voluntários jovens. Dois ensaios clínicos mostraram que o ginseng não altera a farmacocinética e a farmacodinâmica da varfarina (Jiang et al., 2004; 2005); porém, o outro estudo mostrou redução no efeito anticoagulante da varfarina, quando a mesma foi administrada juntamente com o ginseng (Yuan et al., 2004). Como os resultados destes estudos foram contraditórios, recomenda-se a monitoração do paciente, devido a possível redução da eficácia dos anticoagulantes orais pelos medicamentos fitoterápicos à base de ginseng (Plotnikoff et al., 2004; Cheng, 2005; Micromedex, 2006).

\section{Estrogênios}

O uso concomitante de medicamentos fitoterápicos à base de ginseng e estrogênios pode provocar efeitos adversos advindos do aumento da atividade estrogênica, tais como mastalgia e sangramento menstrual excessivo. Alguns relatos de casos sugerem que o ginseng possui atividade semelhante aos hormônios estrogênicos (Palmer et al., 1978; Punnonen \& Lukola, 1980; Greenspan, 1983). Devido a esse possível efeito estrogênico, deve-se evitar o uso de medicamentos à base de ginseng em pacientes com câncer de mama, sangramento vaginal anormal não diagnosticado, tromboflebite ativa, distúrbios tromboembólicos e em gestantes (Micromedex, 2006).

\section{Anti-hipertensivos}

Um ensaio clínico conduzido com 22 voluntários saudáveis avaliou a possível interação do uso concomitante de um medicamento à base de ginseng e nifedipina (vasodilatador antagonista dos canais de cálcio). Durante 18 dias, um grupo foi tratado com $200 \mathrm{mg} /$ dia de um medicamento à base de ginseng e uma dose única de nifedipina $(10 \mathrm{mg})$, enquanto o grupo controle foi tratado somente com a nifedipina. Após $30 \mathrm{~min}$, observou-se um aumento de 53\% na concentração plasmática da nifedipina em relação ao grupo controle. Como conseqüência, verificou-se um aumento significativo nos efeitos adversos desse antihipertensivo, tais como cefaléia, constipação, edema de tornozelos e insuficiência cardíaca (Smith et al., 2001).

Há na literatura, o relato de um caso de uma mulher de 63 anos, portadora de glomerulonefrite membranosa e tratada com furosemida e ciclosporina, que foi hospitalizada com quadro de hipertensão e edema. Estas complicações ocorreram após 10 dias de tratamento com uma preparação contendo ginseng e germânio (mineral presente em suplementos alimentares 
para fortalecimento do sistema imunológico). O quadro foi controlado após a administração intravenosa de 240 mg de furosemida e a suspensão da preparação à base de ginseng. Os autores relataram a possível interação entre o ginseng e a furosemida, mas a presença do germânio pode ter favorecido o aparecimento das complicações, principalmente, pelo fato de que o germânio, quando usado a longo prazo, pode provocar danos renais (Becker et al., 1996).

\section{Hipoglicemiantes}

Alguns estudos sugerem que medicamentos fitoterápicos à base de Panax ginseng ou Panax quinquefolium podem provocar hipoglicemia em pacientes tratados com hipoglicemiantes orais (Vuksan et al., 2000a,b) e, também, podem reduzir a glicemia pós-prandial em indivíduos saudáveis (Vuksan et al., 2000b,c; Vuksan et al., 2001). Os mecanismos desta possível interação medicamentosa ainda não foram elucidados, mas provavelmente, ocorre pelo fato de que, em estudos em animais, o ginseng aumentou a sensibilidade aos receptores da insulina ( $\mathrm{Ng} \&$ Yeung, 1985; Ohnishi et al., 1996) e, também, a sua secreção (Kimura et al., 1981).

\section{Etanol}

Um estudo clínico aberto e não-randomizado, conduzido com voluntários saudáveis, mostrou uma redução de $30 \%$ na concentração plasmática do etanol, quando esse foi administrado juntamente com um extrato de ginseng (Lee et al., 1987). Esta interação pode ser explicada pelo fato de que os ginsenosídeos retardam o esvaziamento gástrico e induzem os sistemas enzimáticos que metabolizam o etanol e, também, a isoforma CYP2E1 (esta isoforma do citocromo P450 hepático é responsável pela metabolização do etanol, juntamente com as enzimas álcool e aldeído desidrogenase e catalase).

\section{Vacinas}

Um ensaio clínico randomizado e multicêntrico avaliou a possível interferência do extrato G115 de ginseng sobre a eficácia de uma vacina polivalente para o tratamento do vírus influenza, em 227 voluntários. Verificou-se uma redução significativa nos sintomas característicos da gripe e, também, um aumento da atividade das células NK sangüíneas nos pacientes tratados com o medicamento fitoterápico (Scaglione et al., 1996). Estes resultados podem ser explicados pelo fato de que o ginseng melhorou a resposta imunológica em animais de laboratório (Hu et al., 2003)

Em suma, os estudos disponíveis sugerem uma potencial interação entre os medicamentos fitoterápicos elaborados com ginseng e antidepressivos inibidores da monoamino oxidade, anti-hipertensivos, contraceptivos à base de estrogênios e hipoglicemiantes orais. Além disso, deve-se monitorar o tempo de pró-trombina nos usuários de ginseng e varfarina.

\section{CONCLUSÃO}

A utilização de plantas medicinais e medicamentos fitoterápicos tem aumentado nos últimos anos, principalmente, pelos portadores de doenças crônicas. Como as plantas medicinais e os medicamentos fitoterápicos são caracterizados por uma mistura complexa de componentes químicos e podem apresentar diversos mecanismos de ação, não há dúvidas de que, quando administrados concomitantemente, podem interagir com diversos fármacos, alterando os seus perfis de eficácia e segurança. Neste estudo, verificouse que as evidências disponíveis sobre a maioria das possíveis interações medicamentosas foram obtidas de estudos in vitro, in vivo ou de relatos de casos. Estes estudos, geralmente, apresentam diversas limitações. Apesar dos estudos in vitro e in vivo serem importantes para a elucidação dos mecanismos envolvidos na interação, geralmente, os seus resultados não podem ser extrapolados para os seres humanos (Ernst 2000a,b). Apesar das informações presentes nos relatos de casos, na maioria das vezes, serem insuficientes, estas fontes de informação são importantes para aumentar as evidências sobre a segurança dos medicamentos fitoterápicos e estimular a condução de estudos clínicos controlados. Mesmo assim, o número de relatos de casos ainda é reduzido, já que não há tradição dos médicos em relacionar os efeitos adversos a medicamentos com prováveis interações com plantas e/ou medicamentos fitoterápicos, dando a idéia, muitas vezes equivocada, de que estes produtos são totalmente seguros. Adicionalmente, muitos pacientes não informam aos profissionais da saúde de que são usuários da fitoterapia e, assim, os casos de interações medicamentosas não podem ser identificados (Hu et al., 2005). No entanto, quando os mesmos chegam a ser identificados, geralmente, são registrados com informações insuficientes, dificultando a conclusão de que a interação foi provocada pelo medicamento fitoterápico e, mais dificilmente, estes relatos são publicados em revistas científicas especializadas. Portanto, recomenda-se que os profissionais da área da saúde documentem os possíveis casos de interações entre medicamentos fitoterápicos e fármacos, contribuindo para aumentar as evidências sobre o perfil de segurança das mesmas. No caso de utilizar fármacos juntamente com tais medicamentos ou mesmo com plantas medicinais, recomenda-se aos usuários solicitar informações aos profissionais da área da saúde, para evitar riscos de interação medicamentosa e prejudicar o seu tratamento. 


\section{REFERÊNCIAS}

Alexandre RFA, Garcia FN, Simões CMO 2005a. Fitoterapia baseada em evidências. Parte 1. Medicamentos fitoterápicos elaborados com ginkgo, hipérico, kava e valeriana. Acta Farma Bonaerense 24: 300-309.

Alexandre RFA, Garcia FN, Simões CMO 2005b. Fitoterapia baseada em evidências. Parte 2. Medicamentos fitoterápicos elaborados com alcachofra, castanha-daíndia, ginseng e maracujá. Acta Farma Bonaerense 24: 310-314.

Atmaca M, Tezcan E, Kuloglu M, Ustundag B, Kirtas O 2005. The effect of extract of ginkgo biloba addition to olanzapine on therapeutic effect and antioxidant enzyme levels in patients with schizophrenia. Psychiatry Clin Neurosci 59: 652-656.

Barnes PM, Powell-Griner E, McFann K, Nahin RL 2004. Complementary and alternative medicine use among adults: United States, 2002. Adv Data 343: 1-19.

Bebbington A, Kulkarni R, Roberts P 2005. Ginkgo biloba: persistent bleeding after total hip arthroplasty caused by herbal self-medication. J Arthroplasty 20: 125-126.

Becker BN, Greene J, Evanson J, Chidsey G, Stone WJ 1996. Ginseng-induced diuretic resistance. JAMA 276: 606-607.

Blumenthal M, Goldberg A, Brinckmann J 2000. Herbal Medicine: Expanded commission e monographs. Austin: American Botanical Council.

Blumenthal M, Ferrier GKL, Cavaliere C 2006. Total sales of herbal supplements in United States show steady growth. HerbalGram 71: 64-66.

Bucci L 2000. Selected herbals and human exercise performance. Am J Clin Nutr 72: 624-636.

Cheng TO 2005. Ginseng and other herbal medicines that interact with warfarin. Int J Cardiol 104: 227.

Cohen AJ, Bartlik B 1998. Ginkgo biloba for antidepressantinduced sexual dysfunction. $J$ Sex Marital Ther 24: 139-143.

Coleman CI, Hebert JH, Reddy P 2003. The effects of Panax ginseng on quality of life. J Clin Pharm Ther 28: 5-15.

Coon JT, Ernst E 2002. Panax ginseng: A systematic review of adverse effects and drug interactions. Drug Safety 25: 323-344.

Cordeiro CHC, Chung MC, Sacramento LVS 2005. Interações medicamentosas de fitoterápicos e fármacos: Hypericum perforatum e Piper methysticum. Rev Bras Farmacogn 15: 272-278.

De Smet PAGM 2005. Herbal medicine: relaxing regulatory standards. $N$ Engl J Med 352: 1176-1178.

DeFeudis FV 1998. Ginkgo biloba extract (EGb 761) From chemistry to the clinic. Wiesbaden: Ullstein Medical.

Drew S, Davies E 2001. Effectiveness of Ginkgo biloba in treating tinnitus: double blind, placebo controlled trial. $B M J$ 322: 1-6.

Ernst E, Willooughby M, Weihmayr TH 1995. Nine possible reasons for choosing complementary medicine. Perfusion 8: 356-358.
Ernst E, Stevinson C 1999. Ginkgo biloba for tinnitus: a review. Clin Otolaryngol Allied Sci 24: 164-7.

Ernst E 2000a. Possible interactions between synthetic and herbal medicinal products Part 1: a systematic review of the indirect evidence. Perfusion 13: 4-15.

Ernst E 2000b. Interactions between synthetic and herbal medicinal products Part 2: a systematic review of the direct evidence. Perfusion 13: 60-70.

Fugh-Berman A 2000. Herb-drug interactions. Lancet 355: 134-138.

Fugh-Bernman A, Ernst E 2001. Herb-drug interactions: review and assessment of report reability. $\mathrm{Br} J \mathrm{Clin}$ Pharmacol 52: 587-595.

Galluzzi S, Zanetti O, Trabucchi M, Frisoni GB 2000. Coma in a patient with Alzheimer's disease taking low-dose trazodone and ginkgo biloba. J Neurol Neurosurg Psychiatry 68: 679-680.

Gerhardt G, Rogalla K, Jaeger J 1990. Drug therapy of disorders of cerebral performance. Randomized comparative study of dihydroergotoxine and Ginkgo biloba extract. Fortschr Med 108: 384-388.

Granger AS 2001. Ginkgo biloba precipitating epileptic seizures. Age Ageing 30: 523-525.

Greenspan EM 1983. Ginseng and vaginal bleeding. JAMA 249:2018.

Gurley BJ, Gardner SF, Hubbard MA, Williams DK, Gentry WB, Khan IA, Shah A 2005. In vivo effects of goldenseal, kava kava, black cohosh, and valerian on human cytochrome P450 1A2, 2D6, 2E1, and 3A4/5 phenotypes. Clin Pharmacol Ther 77: 415-426.

Hartel U, Volger E 2004. Use and acceptance of classical natural and alternative medicine in Germany findings of a representative population-based survey. Forsch Komplementarmed Klass Naturheilkd 11: 327-334.

Hillebrand MD, Oliveira WF 2004. Efeito do EGB 761 (Extrato de Ginkgo biloba) sobre a qualidade de vida em pacientes asmáticos moderados submetidos a tratamento de manutenção. Rev Bras Farmacogn 14(Supl. 1): 24-26.

Hopfenmüller W 1994. Evidence for a therapeutic effect of Ginkgo biloba special extract. Meta-analysis of 11 clinical studies in patients with cerebrovascular insufficiency in old age Arzneimittel-Forsch 44: 1005-1013.

Hu S, Concha C, Lin F, Persson Waller K 2003. Adjuvant effect of ginseng extracts on the immune responses to immunization against Staphylococcus aureus in dairy cattle. Vet Immunol Immunopathol 91: 29-37.

Hu Z, Yang X, Ho PC, Chan SY, Heng PW, Chan E, Duan W, Koh HL, Zhou S 2005. Herb-drug interactions: a literature review. Drugs 65: 1239-1282.

Ionnides C 2002. Pharmacocinetic interactions between herbal remedies and medicinal drugs. Xenobiotica 32: 451-478.

Izzo AA, Ernst E 2001. Interactions between herbal medicines and prescribed drugs: a systematic review. Drugs 61: 2163-2175.

Jacoby D, Mohler ER 2004. Drug treatment of intermittent claudication. Drugs 64: 1657-1670. 
Janetzky K, Morrealy AP 1997. Probable interaction between warfarin and ginseng. Am J Health Syst Pharm 54: 692-693.

Jiang X, Williams KM, Liauw WS, Ammit AJ, Roufogalis BD, Duke CC, Day RO, McLachlan AJ 2004. Effect of St John's wort and ginseng on the pharmacokinetics and pharmacodynamics of warfarin in healthy subjects. Br J Clin Pharmacol 57: 592-9.

Jiang X, Williams KM, Liauw WS, Ammit AJ, Roufogalis BD, Duke CC, Day RO, McLachlan AJ 2005. Effect of ginkgo and ginger on the pharmacokinetics and pharmacodynamics of warfarin in healthy subjects. Br J Clin Pharmacol 59: 425-32.

Jones BD, Runikis AM 1987. Interactions of ginseng with phenelzine. J Clin Psychopharmacol 7: 201-202.

Kang BJ, Lee SJ, Kim MD, Cho MJ 2002. A placebocontrolled, double-blind trial of Ginkgo biloba for antidepressant-induced sexual dysfunction. Hum Psychopharmacol 17: 279-84.

Kim HJ, Chum YJ, Park JD, Kim SI, Roh JK, Jeong TC 1997. Protection of rat liver microsomes against carbon tethracloride-induced lipid peroxidation by red ginseng saponin through cytochrome $\mathrm{P} 450$ inhibition. Planta Med 63: 415-418.

Kimura M, Waki I, Chujo T, Kikuchi T, Hiyama C, Yamazaki K, Tanaka O 1981. Effects of hypoglycemic components in ginseng radix on blood insulin level in alloxan diabetic mice and on insulin release from perfused rat pancreas. J Pharmacobiodyn 4: 410-7.

Kleijnen J, Knipschild P 1992. Ginkgo biloba for cerebral insufficiency. Br J Clin Pharmacol 34: 352-358.

Lambrecht JE, Hamilton W, Rabinovich A 2000. A review of herb-drug interations: documented and theoretical. US Pharma 25: 8

Le Bars PL, Velasco FM, Ferguson JM, Dessain EC, Kieser M 2002. Influence of the severity of cognitive impairment on the effect of the Ginkgo biloba extract EGB $761^{\circledR}$ in Alzheimer's disease. Neuropsychobiology 45: 19-26.

Lee FC, Ko JH, Park JK, Lee JS 1987. Effects of Panax ginseng on blood alcohol clearance in man. Clin Exp Pharmacol Physiol 14: 543-546.

Liu CX, Xiao PG 1992. Recent advances on ginseng research in China. J Ethnopharmacol 36: 27-38.

MacLennan AH, Wilson DH, Taylor AW 1996. Prevalence and cost of alternative medicine in Australia. Lancet 347: 569-573.

Markowitz JS, Donovan JL, Lindsay DeVane C, Sipkes L, Chavin KD 2003.Multiple-dose administration of Ginkgo biloba did not affect cytochrome P-450 2D6 or 3A4 activity in normal volunteers. J Clin Psychopharmacol 23: 576-81.

Matthews MK 1998. Association of Ginkgo biloba whit intracerebral hemorrhage. Neurolog 50: 1933-1934.

Meisel C, Johne A, Roots I 2003. Fatal intracerebral mass bleeding associated with Ginkgo biloba and ibuprofen. Atherosclerosis 167: 367.

Menniti-Ippolito F, Gargiulo L, Bologna E, Forcella E, Raschetti R 2002. Use of unconventional medicine in Italy: a nation-wide survey. Eur J Clin Pharmacol
58: 61-64.

Micromedex 2005. Drug-Reax Interactive Drug interactions 2005: Ginkgo. http://cdrompro.com.br/micromedex/ trial.html, acessada em abril de 2005.

Micromedex 2006. Drug-Reax Interactive Drug Interactions: Ginseng 2006. http://cdrompro.com.br/micromedex/ trial.html, acessada em: 27 de outubro de 2006.

Mills E, Wu P, Johnston BC, Gallicano K, Clarke M, Guyatt G 2005. Natural health product-drug interactions: a systematic review of clinical trials. Ther Drug Monit 27: 549-557.

Momesso JC 2002. Análise do mercado nacional de fitoterápicos: Quais os passos, principais avanços, descobertas, capital movimentado e faturamento das indústrias de fitoterápicos. Palestra proferida em 27 de maio de 2002, São Paulo, SP, promovida pelo Institute for International Research do Brasil LTDA sobre o mercado de fitoterápicos.

Morgenstern C, Biermann E 2002. The efficacy of ginkgo special extract EGb 761 in patients with tinnitus. Int J Clin Pharmacol Ther 40: 188-197.

$\mathrm{Ng}$ TB, Yeung HW 1985. Hypoglycemic constituents of Panax ginseng. Gen Pharmacol 16: 549-552.

Ohnishi Y, Takagi S, Miura T, Usami M, Kako M, Ishihara E, Yano H, Tanigawa K, Seino Y 1996. Effect of ginseng radix on GLUT2 protein content in mouse liver in normal and epinephrine-induced hyperglycemic mice. Biol Pharm Bull 19: 1238-1240.

Oken BS, Storzbach DM, Kaye JA 1998. The efficacy of Ginkgo biloba on cognitive function in Alzheimer disease. Arch Neurol 55: 1409-1415.

Palmer BV, Montgomery ACV, Monteiro JCMP 1978. Ginseng and mastalgia. BMJ 1: 1284.

Pittler MH, Ernst E 2000. Ginkgo biloba extract for the treatment of intermittent claudication: a meta-analysis of randomized trials. Am J Med 108: 276-281.

Plotnikoff GA, McKenna D, Watanabe K, Blumenthal M 2004. Ginseng and warfarin interactions. Ann Intern Med 141: 893-894.

Prehn JH, Krieglstein J 1993. Platelet-activating factor antagonists reduce excitotoxic damage in cultured neurons from embryonic chick telencephalon and protect the rat hippocampus and neocortex from ischemic injury in vivo. $J$ Neurosci Res 34: 179-188.

Punnonen R, Lukola A 1980. Estrogen-like effect of ginseng. Lancet 181: 1110.

Rejali D, Sivakumar A, Balaji N 2004. Ginkgo biloba does not benefit patients with tinnitus: a randomized placebocontrolled double-blind trial and meta-analysis of randomized trials. Clin Otolaryngol Allied Sci 29: 226-231.

Ribeiro AQ, Leite JPV, Dantas-Barros AM 2005. Perfil de utilização de fitoterápicos em farmácias comunitárias de Belo Horizonte sob a influência da legislação nacional. Rev Bras Farmacogn 15: 65-70.

Rogers SL, Farlow MR, Dooby RS, Mohs R, Friedhoff LT 1998. A 24-week, double-blind placebo-controlled trial of donezepil in patients with Alzheimer's disease. Neurology 50: 136-145.

Rosenblatt M, Mindel J 1997. Spontenous bilateral hyphema 
associated with ingestion of Ginkgo biloba extract. $N$ Engl J Med 336: 1108.

Sasaki K, Hatta S, Haga M, Ohshika H 1999. Effects of bilobalide on gamma-aminobutyric acid levels and glutamic acid decarboxylase in mouse brain. Eur $J$ Pharmacol 367: 165-173.

Scaglione F, Cattaneo G, Alessandria M, Cogo R 1996. Efficacy and safety of the standardised Ginseng extract G115 for potentiating vaccination against the influenza syndrome and protection against the common cold. Drugs Exp Clin Res 22: 65-72.

Schulz V 2003. Ginkgo extract or cholinesterase inhibitors in patients with dementia: what clinical trials and guidelines fail to consider. Phytomedicine 10: 74-79.

Shader RI, Greenblatt DJ 1985. Bees, ginseng and MAOIs revisited. J Clin Psychopharmacol 8: 235.

Shader RI, Greenblatt DJ 1988. Phenelzina and the dream machine-ramblings and reflections. $J$ Clin Psychopharmacol 5: 65.

Silva MIG, Gondim APS, Nunes IFS, Sousa FCF 2006. Utilização de fitoterápicos nas unidades básicas de atenção à saúde da família no município de Maracanaú (CE). Rev Bras Farmacogn 16: 455-462.

Simões CMO, Mentz LA, Schenkel EP, Irgang BE, Stehmann JR 1986. Plantas da medicina popular no Rio Grande do Sul Porto Alegre: Editora da Universidade/ UFRGS.

Smith M, Lin KM, Zheng YP 2001. PIII-89 an open trial of nifedipine-herb interactions: nifedipine with St John's wort, ginseng or ginkgo biloba. Clin Pharmacol Ther 69: 86.

Smith PF, Zheng Y, Darlington CL 2005. Ginkgo biloba extracts for tinnitus: More hype than hope? J Ethnopharmacol 100: 95-99.

Sparreboom A, Cox MC, Acharya MR, Figg WD 2004. Herbal remedies in the United States: potential adverse interactions with anticancer agents. J Clin Oncol 22: 2489-2503.

van den Bout-van den Beukel CJ, Koopmans PP, van der Ven AJ, De Smet PA, Burger DM 2006. Possible drugmetabolism interactions of medicinal herbs with antiretroviral agents. Drug Metab Rev 38: 477-514.

Varona FJC, Morales MPA 2005. Ginkgo biloba y hemorragia cerebral. An Med Interna 22: 199.

Vogler BK, Pittler MH, Ernst E 1999. The efficacy of ginseng. A systematic review of randomised clinical trials. Eur J Clin Pharmacol 55: 567-575, 1999.

Vuksan V, Stavro MP, Sievenpiper JL, Beljan-Zdravkovic U, Leiter LA, Josse RG, Xu Z 2000a. Similar postprandial glycemic reductions with escalation of dose and administration time of American ginseng in type 2 diabetes. Diabetes Care 23: 1221-1226.

Vuksan V, Sievenpiper JL, Koo VY, Francis T, BeljanZdravkovic U, Xu Z, Vidgen E 2000b. American ginseng (Panax quinquefolius $\mathrm{L}$ ) reduces postprandial glycemia in nondiabetic subjects and subjects with type 2 diabetes mellitus. Arch Intern Med 160: 1009-13.

Vuksan V, Stavro MP, Sievenpiper JL, Koo VY, Wong E,
Beljan-Zdravkovic U, Francis T, Jenkins AL, Leiter LA, Josse RG, $\mathrm{Xu} \mathrm{Z} \mathrm{2000c.American} \mathrm{ginseng}$ improves glycemia in individuals with normal glucose tolerance:effect of dose and time escalation. J Am Coll Nutr 19: 738-744.

Vuksan V, Sievenpiper JL, Wong J, Xu Z, Beljan-Zdravkovic U, Arnason JT, Assinewe V, Stavro MP, Jenkins AL, Leiter LA, Francis T 2001. American ginseng (Panax quinquefolius $\mathrm{L}$.) attenuates postprandial glycemia in a time-dependent but not dose-dependent manner in healthy individuals. Am J Clin Nutr 73: 753-8.

Wettstein A 2000. Cholisterase inhibitors and ginkgo extracts - are they comparable in the treatment of dementia? Comparasion of publish placebo-controlled efficacy studies of at least six month's duration. Phytomedicine 6: 393-401.

Wheatley D 2004. Triple-blind, placebo-controlled trial of Ginkgo biloba in sexual dysfunction due to antidepressant drugs. Hum Psychopharmacol 19: 545-548.

Williamson EM 2005. Interactions between herbal and conventional medicines 2005. Expert Opin Drug Saf 4: $355-378$

Yin OQ, Tomlinson B, Waye MM, Chow AH, Chow MS 2004. Pharmacogenetics and herb-drug interactions: experience with Ginkgo biloba and omeprazole. Pharmacogenetics 14: 841-850.

Yoshioka M, Ohnishi N, Koishi T, Obata Y, Nakagawa M, Matsumoto T, Tagagi K, Takara K, Ohkuni T, Yokoyama T, Kuroda K 2004. Studies on interactions between functional foods or dietary supplements and medicines. IV. Effects of Ginkgo biloba leaf estract on the pharmacokinetics and pharmacodynamics of nifedipine in healthy volunteers. Biol Pharm Bull 27: 2006-2009.

Yuan CS, Wei G, Dey L, Karrison T, Nahlik L, Maleckar S, Kasza K, Ang-Lee M, Moss J 2004. Brief communication: American ginseng reduces warfarin's effect in healthy patients: a randomized, controlled Trial. Ann Intern Med 14: 23-27.

Zhang XY, Zhou DF, Su JM, Zhang PY 2001a. The effect of extract of Ginkgo biloba added to haloperidol on superoxide dismutase in inpatients with chronic schizophrenia. J Clin Psychopharmacol 21: 85-8.

Zhang XY, Zhou DF, Zhang PY, Wu GY, Su JM, Cao LY 2001b. A double-blind, placebo-controlled trial of extract of Ginkgo biloba added to haloperidol in treatment-resistant patients with schizophrenia. $J$ Clin Psychiatry 62: 878-883.

Zhou D, Zhang X, Su J, Nan Z, Cui Y, Liu J, Guan Z, Zhang P, Shen Y 1999. The effects of classic antipsychotic haloperidol plus the extract of Ginkgo biloba on superoxide dismutase in patients with chronic refractory schizophrenia. Chin Med J 112: 1093-1096.

Zhu M, Chan KW, NG LS, Chang Q, Chang S, Li RC 1999. Possible influences of ginseng on the pharmacokinetics and pharmacodynamics of warfarin in rats. J Pharm Pharmacol 51: 175-180. 